\title{
Calculation of hyperfine structure constants of small molecules using Z-vector method in the relativistic coupled-cluster framework
}

\author{
SUDIP SASMAL $^{\mathrm{a}}$, KAUSHIK TALUKDAR ${ }^{\mathrm{b}}$, MALAYA K NAYAK $^{\mathrm{c}}$, NAYANA VAVAL ${ }^{\mathrm{a}}$ and \\ SOURAV PAL ${ }^{\mathrm{b}, *}$ \\ ${ }^{a}$ Electronic Structure Theory Group, Physical Chemistry Division, CSIR-National Chemical Laboratory, \\ Pune, Maharashtra 411 008, India \\ ${ }^{b}$ Department of Chemistry, Indian Institute of Technology Bombay, Powai, Mumbai, Maharashtra 400 076, India \\ ${ }^{c}$ Theoretical Chemistry Section, Bhabha Atomic Research Centre, Trombay, Mumbai, Maharashtra 400 085, India \\ e-mail: s.pal@ncl.res.in
}

MS received 2 September 2016; accepted 8 September 2016

\begin{abstract}
The Z-vector method in the relativistic coupled-cluster framework is employed to calculate the parallel and perpendicular components of the magnetic hyperfine structure constant of a few small alkaline earth hydrides $(\mathrm{BeH}, \mathrm{MgH}$, and $\mathrm{CaH})$ and fluorides $(\mathrm{MgF}$ and $\mathrm{CaF})$. We have compared our Z-vector results with the values calculated by the extended coupled-cluster (ECC) method reported in Phys. Rev. A 91022512 (2015). All these results are compared with the available experimental values. The Z-vector results are found to be in better agreement with the experimental values than those of the ECC values.
\end{abstract}

\section{Introduction}

The precise calculation of the wavefunction of a manyelectron system is extremely important to obtain accurate result of different atomic and molecular properties. For the calculation of various properties like hyperfine structure $(\mathrm{HFS})$ constants, and various parity $(\mathcal{P})$ and time reversal $(\mathcal{T})$-violating interaction constants (like effective electric field $\left(E_{\text {eff }}\right)$ experienced by the unpaired electron and scalar-pseudoscalar (S-PS) $\mathcal{P}, \mathcal{T}$ odd interaction constant $\left(W_{\mathrm{s}}\right)$ ), an accurate wavefunction is needed in the near nuclear region. The calculation of these types of $\mathcal{P}, \mathcal{T}$-odd properties is very important because they cannot be measured by any experimental technique and are very important to unravel many mysteries of physics. ${ }^{1-7}$ The accuracy of these types of properties can be accessed by comparing theoretically obtained HFS constants with available experimental values. Light hetero-diatomic molecules are very useful for testing the accuracy of wavefunction in the near nuclear region as the magnetic moment of both nuclei interacts with the unpaired electron to produce HFS splitting. ${ }^{8}$

The accurate calculation of HFS needs to include both relativistic and electron correlation effects in an intertwine manner. The Dirac-Hartree-Fock (DHF) method is the most elegant way to include the relativistic effects in a single determinant theory. The dynamic

*For correspondence

Celebrating 100 years of Lewis Chemical Bond correlation of opposite spin electrons which is missed in DHF theory can be included by single reference coupled cluster (SRCC) method. ${ }^{9}, 10$ The SRCC equations can be solved either by variational (VCC) or non-variational (NCC) way. In NCC, the property calculation can be done either in the expectation value or in the energy derivative method. In general, these two methods produce two different results as the NCC is non-variational and thus, does not satisfy the generalized Hellmann-Feynman (GHF) theorem. ${ }^{11,12}$ However, the energy derivative method is superior to the expectation value method because the first-order energy derivative is the corresponding expectation value plus some additional terms, which makes the derivative approach closer to full configuration interaction property value. The expectation value in NCC also produces a nonterminating series and one needs to use some truncation scheme for practical purpose. This introduces an additional error.

On the other hand, VCC satisfies the GHF theorem and thus, both expectation value and derivative methods produce identical results. Among many VCC, ${ }^{13}$ extended coupled-cluster (ECC), ${ }^{14,15}$ expectation value coupled-cluster (XCC), ${ }^{16,17}$ and unitary coupled-cluster $(\mathrm{UCC})^{18-23}$ are the most familiar in literature. Recently, we implemented ECC in four-component relativistic framework to calculate the first-order properties and we applied this to calculate the magnetic HFS constants of atoms and molecules. ${ }^{24}$ In ECC, the amplitude equations for both excitation and deexcitation operators 
are coupled and thus, we have to solve them simultaneously. In the Z-vector method (an energy derivative method in NCC), ${ }^{25,26}$ the equations for excitation operators are decoupled from the equations of deexcitation operators. This accelerates the convergence in Z-vector method and saves enormous computational time.

In this paper, we have calculated parallel and perpendicular magnetic HFS constants of $\mathrm{BeH}, \mathrm{MgH}, \mathrm{CaH}$, $\mathrm{MgF}$, and $\mathrm{CaF}$ molecules using the $\mathrm{Z}$-vector coupledcluster method and compared them with the experimental results wherever available. We also compared our results with the ECC results taken from Ref. ${ }^{24}$

The manuscript is organized as follows. A brief overview of the $\mathrm{Z}$-vector method including concise details of parallel and perpendicular components of the magnetic HFS constant is described in Sec. 2. Computational details are given in Sec. 2.3. We presented our calculated results and discussed about those in Sec. 3 before making the final remark in Sec. 4. Atomic unit is used consistently unless stated otherwise.

\section{Theory and Computational Details}

\subsection{Z-vector method}

In a single determinant theory, the DHF method yields the best description of the ground state and for this reason, it is used as a reference state for the treatment of missing electron correlation. The Dirac-Coulomb (DC) Hamiltonian is used in this calculation and can be expressed as

$$
\begin{aligned}
H_{D C}= & \sum_{i}\left[-c(\vec{\alpha} \cdot \vec{\nabla})_{i}+\left(\beta-\mathbb{1}_{4}\right) c^{2}+V^{n u c}\left(r_{i}\right)\right. \\
& \left.+\sum_{j>i} \frac{1}{r_{i j}} \mathbb{1}_{4}\right],
\end{aligned}
$$

where, $\alpha$ and $\beta$ are the usual Dirac matrices, $c$ is the speed of light, $\mathbb{1}_{4}$ is the $4 \times 4$ identity matrix. $V^{n u c}\left(r_{i}\right)$ is the nuclear potential term and we have used Gaussian charge distribution for this purpose. The DHF method transforms the complicated many-electron problem into many one-electron problems and in this process misses the correlation between opposite spin electrons. In this work, the missing dynamic correlation is incorporated by using the SRCC method. The SRCC wavefunction is given by

$$
\left|\Psi_{c c}\right\rangle=e^{T}\left|\Phi_{0}\right\rangle
$$

where $\Phi_{0}$ is the DHF wavefunction and $T$ is the coupled-cluster excitation operator which is given by

$$
T=T_{1}+T_{2}+\cdots+T_{N}=\sum_{n}^{N} T_{n},
$$

with

$$
T_{m}=\frac{1}{(m !)^{2}} \sum_{i j \ldots a b \ldots} t_{i j \ldots}^{a b \ldots} a_{a}^{\dagger} a_{b}^{\dagger} \ldots a_{j} a_{i},
$$

where $i, j$ are the hole and $a, b$ are the particle indices. $t_{i j . .}^{a b .}$ are the cluster amplitudes corresponding to the cluster operator $T_{m}$. The equations for n-body cluster amplitudes are given as

$$
\left\langle\Phi_{i . .}^{a . .}\left|\left(H_{N} e^{T}\right)_{c}\right| \Phi_{0}\right\rangle=0,
$$

where $\mathrm{H}_{N}$ is the normal ordered DC Hamiltonian and subscript $c$ means only the connected terms exist in the contraction between $\mathrm{H}_{N}$ and $\mathrm{T}$. In the coupled-cluster single and double (CCSD) model, $T=T_{1}+T_{2}$. The explicit equations for $\mathrm{T}_{1}$ and $\mathrm{T}_{2}$ are given as

$$
\left\langle\Phi_{i}^{a}\left|\left(H_{N} e^{T}\right)_{c}\right| \Phi_{0}\right\rangle=0,\left\langle\Phi_{i j}^{a b}\left|\left(H_{N} e^{T}\right)_{c}\right| \Phi_{0}\right\rangle=0 .
$$

Once the $\mathrm{T}_{1}$ and $\mathrm{T}_{2}$ amplitudes are solved, the correlation energy can be obtained as

$$
E^{c o r r}=\left\langle\Phi_{0}\left|\left(H_{N} e^{T}\right)_{c}\right| \Phi_{0}\right\rangle .
$$

The SRCC solved in this way is non-variational in nature and thus, the SRCC energy is not optimized with respect to the molecular orbital coefficients $\left(\mathrm{C}_{M}\right)$ and the determinantal coefficients $\left(\mathrm{C}_{D}\right)$ in the expansion of the correlated many-electron wavefunction for a fixed nuclear geometry. ${ }^{11}$ Therefore, for the energy derivative calculation, one needs to incorporate the derivative of energy with respect to $\mathrm{C}_{M}$ and $\mathrm{C}_{D}$ in addition to the derivative of these two coefficients with respect to the external field parameter. However, the derivative terms related to $\mathrm{C}_{D}$ and $\mathrm{C}_{M}$ can be integrated by solving linear equations for each coefficient where the solutions are in general, perturbation dependent. This means, for each type of properties, one needs to solve a different set of linear equations. However, in Z-vector method, this can be avoided and the derivative terms related to $\mathrm{C}_{D}$ can be incorporated by the introduction of a perturbation independent operator, $\Lambda .{ }^{26}$ The antisymmetrized form of the $\Lambda$ operator is given as

$$
\Lambda=\Lambda_{1}+\Lambda_{2}+\ldots+\Lambda_{N}=\sum_{n}^{N} \Lambda_{n},
$$

where

$$
\Lambda_{m}=\frac{1}{(m !)^{2}} \sum_{i j . a b . .} \lambda_{a b . .}^{i j . .} a_{i}^{\dagger} a_{j}^{\dagger} \ldots . a_{b} a_{a},
$$

where $i, j$ are the hole and $a, b$ are the particle indices. $\lambda_{a b . .}^{i j .}$ are the cluster amplitudes corresponding to the cluster operator $\Lambda_{m}$. From the form of the $\Lambda$ operator, 
Table 1. Parallel $\left(A_{\|}\right)$and perpendicular $\left(A_{\perp}\right)$ magnetic hyperfine structure constant of molecules in MHz.

\begin{tabular}{|c|c|c|c|c|c|c|c|c|c|c|c|}
\hline \multirow[b]{3}{*}{ Molecule } & \multirow[b]{3}{*}{ Atom } & \multicolumn{5}{|c|}{$\mathrm{A}_{\|}$} & \multicolumn{5}{|c|}{$\mathrm{A}_{\perp}$} \\
\hline & & \multirow[b]{2}{*}{$\mathrm{ECC}$} & \multirow[b]{2}{*}{ Z-vector } & \multirow[b]{2}{*}{ Expt. ${ }^{34}$} & \multicolumn{2}{|r|}{$\delta \%$} & \multirow[b]{2}{*}{$\mathrm{ECC}$} & \multirow[b]{2}{*}{ Z-vector } & \multirow[b]{2}{*}{ Expt. ${ }^{34}$} & \multicolumn{2}{|c|}{$\delta \%$} \\
\hline & & & & & $\mathrm{ECC}$ & Z-vector & & & & $\mathrm{ECC}$ & Z-vector \\
\hline \multirow[t]{2}{*}{$\mathrm{BeH}$} & ${ }^{1} \mathrm{H}$ & 204.1 & 199.5 & $201(1)^{8}$ & 1.5 & 0.8 & 185.6 & 181.0 & $190.8(3)^{8}$ & 2.8 & 5.4 \\
\hline & ${ }^{9} \mathrm{Be}$ & -200.6 & -201.3 & $-208(1)^{8}$ & 3.7 & 3.3 & -186.0 & -186.8 & $-194.8(3)^{8}$ & 4.7 & 4.3 \\
\hline \multirow[t]{2}{*}{$\mathrm{MgH}$} & ${ }^{1} \mathrm{H}$ & & 276.2 & $298(1)^{35}$ & & 7.9 & & 271.1 & $264(1)^{35}$ & & 2.6 \\
\hline & ${ }^{25} \mathrm{Mg}$ & & -188.1 & $-226(3)^{35}$ & & 20.1 & & -172.0 & $-218(1)^{35}$ & & 26.7 \\
\hline \multirow[t]{2}{*}{$\mathrm{CaH}$} & ${ }^{1} \mathrm{H}$ & 146.4 & 143.7 & $138(1)^{35}$ & 5.7 & 4.0 & 141.9 & 139.0 & $134(1)^{35}$ & 5.6 & 3.6 \\
\hline & ${ }^{43} \mathrm{Ca}$ & -321.6 & -331.2 & & & & -295.7 & -305.2 & & & \\
\hline \multirow[t]{2}{*}{$\mathrm{MgF}$} & ${ }^{19} \mathrm{~F}$ & 320.9 & 322.6 & $331(3)^{36}$ & 3.1 & 2.6 & 153.3 & 148.7 & $143(3)^{36}$ & 6.7 & 3.8 \\
\hline & ${ }^{25} \mathrm{Mg}$ & -282.6 & -281.8 & & & & -270.4 & -269.6 & & & \\
\hline \multirow[t]{2}{*}{$\mathrm{CaF}$} & ${ }^{19} \mathrm{~F}$ & & 131.7 & $149(3)^{36}$ & & 13.1 & & 83.8 & $106(3)^{36}$ & & 26.5 \\
\hline & ${ }^{43} \mathrm{Ca}$ & & -426.4 & & & & & -408.9 & & & \\
\hline
\end{tabular}

it is clear that it is a de-excitation operator. The $\Lambda$ amplitude equation is given by

$$
\begin{gathered}
\left\langle\Phi_{0}\left|\left[\Lambda\left(H_{N} e^{T}\right)_{c}\right]_{c}\right| \Phi_{i . .}^{a . .}\right\rangle+\left\langle\Phi_{0}\left|\left(H_{N} e^{T}\right)_{c}\right| \Phi_{i . .}^{a . .}\right\rangle \\
+\left\langle\Phi_{0}\left|\left(H_{N} e^{T}\right)_{c}\right| \Phi_{i n t}\right\rangle\left\langle\Phi_{i n t}|\Lambda| \Phi_{i . .}^{a . .}\right\rangle=0,
\end{gathered}
$$

where $\Phi_{i n t}$ is the determinant corresponding to the intermediate excitation between $\Phi_{0}$ and $\Phi_{i . . .}^{a . .}$ In CCSD model, $\Lambda=\Lambda_{1}+\Lambda_{2}$ and the explicit equations for the amplitudes of $\Lambda_{1}$ and $\Lambda_{2}$ operators are given by

$$
\begin{gathered}
\left\langle\Phi_{0}\left|\left[\Lambda\left(H_{N} e^{T}\right)_{c}\right]_{c}\right| \Phi_{i}^{a}\right\rangle+\left\langle\Phi_{0}\left|\left(H_{N} e^{T}\right)_{c}\right| \Phi_{i}^{a}\right\rangle=0, \\
\left\langle\Phi_{0}\left|\left[\Lambda\left(H_{N} e^{T}\right)_{c}\right]_{c}\right| \Phi_{i j}^{a b}\right\rangle+\left\langle\Phi_{0}\left|\left(H_{N} e^{T}\right)_{c}\right| \Phi_{i j}^{a b}\right\rangle \\
+\left\langle\Phi_{0}\left|\left(H_{N} e^{T}\right)_{c}\right| \Phi_{i}^{a}\right\rangle\left\langle\Phi_{i}^{a}|\Lambda| \Phi_{i j}^{a b}\right\rangle=0 .
\end{gathered}
$$

Finally, the energy derivative can be given as

$$
\Delta E^{\prime}=\left\langle\Phi_{0}\left|\left(O_{N} e^{T}\right)_{c}\right| \Phi_{0}\right\rangle+\left\langle\Phi_{0}\left|\left[\Lambda\left(O_{N} e^{T}\right)_{c}\right]_{c}\right| \Phi_{0}\right\rangle,
$$

where, $O_{N}$ is the derivative of the normal ordered perturbed Hamiltonian with respect to the external field of perturbation.

\subsection{Magnetic hyperfine structure constants}

The interaction of the electromagnetic field generated by electrons with the magnetic dipole moment of the nucleus is responsible for the magnetic HFS. ${ }^{27}$ The magnetic vector potential $(\vec{A})$ due to the magnetic moment $\left(\vec{\mu}_{k}\right)$ of a nucleus is given as

$$
\vec{A}=\frac{\vec{\mu}_{k} \times \vec{r}}{r^{3}} \text {. }
$$

The perturbed HFS Hamiltonian of an atom due to $\vec{A}$ in the Dirac theory is given by

$$
H_{h y p}=\sum_{i}^{n} \vec{\alpha}_{i} \cdot \vec{A}_{i},
$$

where $n$ is the total no of electrons and $\alpha_{i}$ denotes the Dirac $\alpha$ matrices for the $\mathrm{i}^{\text {th }}$ electron. For a diatomic molecule, the $\mathrm{z}$ (along the molecular axis) and $\mathrm{x}$ or $y$ (i.e., perpendicular to molecular axis) projection of the expectation value of the corresponding perturbed HFS Hamiltonian gives the parallel $\left(A_{\|}\right)$and perpendicular $\left(A_{\perp}\right)$ magnetic HFS constant, respectively. The expression for $A_{\|}$and $A_{\perp}$ can be written as

$$
A_{\|(\perp)}=\frac{\vec{\mu}_{k}}{I \Omega} \cdot\left\langle\Psi_{\Omega}\left|\sum_{i}^{n}\left(\frac{\vec{\alpha}_{i} \times \vec{r}_{i}}{r_{i}^{3}}\right)_{z(x / y)}\right| \Psi_{\Omega(-\Omega)}\right\rangle,
$$

where $I$ is the nuclear spin quantum number and $\Omega$ represents the $\mathrm{z}$ component (along molecular axis) of the total angular momentum of the diatomic molecule.

\subsection{Computational details}

We have used the locally modified version of DIRAC $10^{28}$ program package to solve the DHF Hamiltonian and to construct the one-electron and two-electron integrals and the HFS integrals. Gaussian charge distribution is considered as nuclear model where the nuclear parameters $^{29}$ are taken as default values of DIRAC10 program package. The basis functions are represented in scalar basis and restricted kinetic balance (RKB) ${ }^{30}$ condition is applied to generate the small component basis functions from the large component basis functions. The unphysical solutions are removed by diagonalizing the free particle Hamiltonian. This generates equal number of positronic and electronic orbital. In our molecular calculations, we have used aug-cc$\mathrm{pCV} \mathrm{Z}^{31}$ for $\mathrm{H}$ atom and aug-cc-pCVQZ ${ }^{31}$ for $\mathrm{F}, \mathrm{Be}$, and $\mathrm{Mg}$ atoms and dyall.cv $4 \mathrm{z}^{32}$ basis for $\mathrm{Ca}$ atom. All the generated orbitals are taken for the correlation calculation of $\mathrm{BeH}$. The cutoff used for the correlation calculations of $\mathrm{MgH}, \mathrm{MgF}, \mathrm{CaH}$, and $\mathrm{CaF}$ molecules 
are 10 a.u., 10 a.u., 15 a.u., and 15 a.u., respectively. The bond-length used for the $\mathrm{BeH}, \mathrm{MgH}, \mathrm{CaH}, \mathrm{MgF}$, and $\mathrm{CaF}$ molecules are $1.343 \AA, 1.7297 \AA, 2.003 \AA$, $1.750 \AA$, and $1.976 \AA$, respectively. ${ }^{33}$

\section{Results and Discussion}

The aim of our present study is to show that the Z-vector method can produce a very good quality wavefunction in the near nuclear region of small molecules and for this purpose we have calculated the HFS constants of some small molecules. In Table 1 , we present the $\mathrm{A}_{\|}$and $\mathrm{A}_{\perp}$ HFS constants of $\mathrm{BeH}, \mathrm{MgH}, \mathrm{CaH}, \mathrm{MgF}$, and $\mathrm{CaF}$ using the $\mathrm{Z}$-vector method in the relativistic coupledcluster framework. The HFS constants of $\mathrm{BeH}, \mathrm{MgF}$ and $\mathrm{CaH}$ using the $\mathrm{Z}$-vector method are also compared with the values obtained in the ECC method reported in Ref. ${ }^{24}$ For this reason, we have taken the identical basis and cutoff for $\mathrm{BeH}, \mathrm{MgF}$ and $\mathrm{CaH}$ as reported in Ref. ${ }^{24}$ These values are compared with the available experimental values ${ }^{8,34-36}$ and the deviations are presented as $\delta \%$. From Table 1 , it is clear that our $\mathrm{Z}$-vector results are in close agreement with the experimental values. The highest and lowest deviations of $\mathrm{Z}$-vector values of $\mathrm{A}_{\|}$occur for ${ }^{25} \mathrm{Mg}$ of $\mathrm{MgH}$ and ${ }^{1} \mathrm{H}$ of $\mathrm{BeH}$, respectively, where the deviations are $37.9 \mathrm{MHz}$ and $1.5 \mathrm{MHz}$, respectively. For the Z-vector results of $\mathrm{A}_{\perp}$, the highest and lowest deviations appear for ${ }^{25} \mathrm{Mg}$ of $\mathrm{MgH}$ and ${ }^{1} \mathrm{H}$ of $\mathrm{MgH}$, respectively, where the deviations are $46 \mathrm{MHz}$ and $7 \mathrm{MHz}$, respectively. From the above observation, we can comment that the Z-vector method can produce very good quality wavefunction in the nuclear region of small molecules. In Figures 1 and 2, we have compared the relative deviations of $\mathrm{A}_{\|}$ and $\mathrm{A}_{\perp}$ values, respectively, between $\mathrm{ECC}$ and $\mathrm{Z}$-vector method. From these figures, it is clear that the Z-vector method always produces better results than the ECC

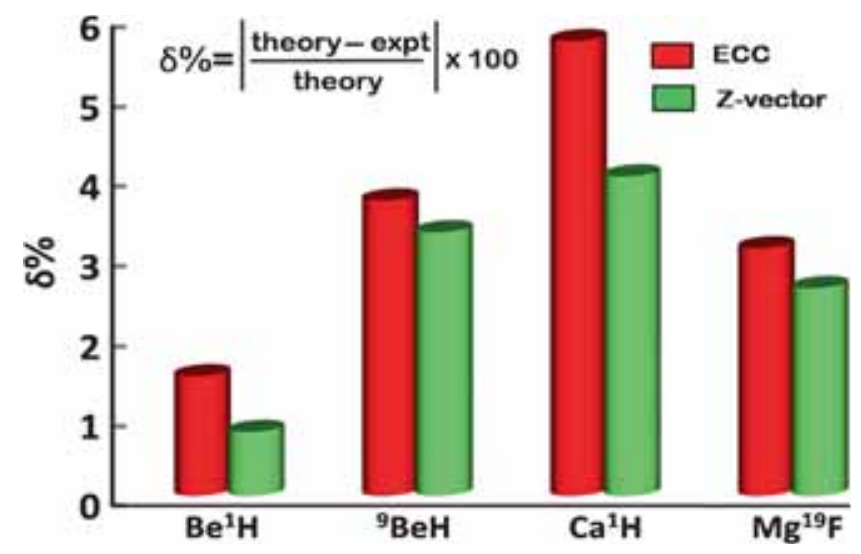

Figure 1. Comparison of relative deviations of $A_{\|}$values between ECC and Z-vector method.

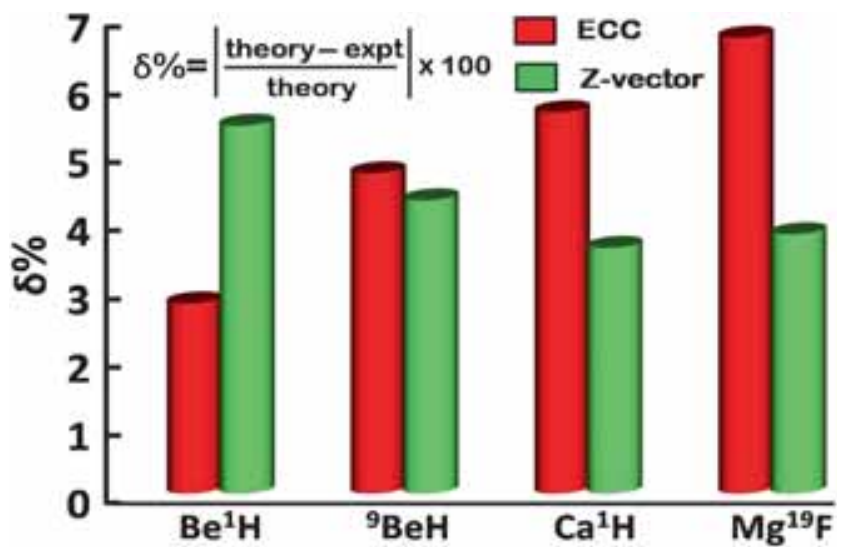

Figure 2. Comparison of relative deviations of $\mathrm{A}_{\perp}$ values between ECC and Z-vector method.

method. The only exception is for $\mathrm{A}_{\perp}$ value of ${ }^{1} \mathrm{H}$ in $\mathrm{BeH}$ where the difference between ECC and Z-vector values is only $4.6 \mathrm{MHz}$. Although the ECC functional generates a terminating series, in the CCSD approximation, the natural truncation produces some computationally very costly terms. To avoid this, the ECC functional in Ref. ${ }^{24}$ is truncated in such a way that either the left vector or the right vector is linear in a double linked form. This introduces some additional errors, which may be the reason for the poor performance of the ECC method compared to the Z-vector method.

\section{Conclusions}

In conclusion, we have applied the $\mathrm{Z}$-vector method in the relativistic coupled-cluster framework to calculate the parallel and perpendicular components of the magnetic HFS constant of small diatomic molecules. Our results show that the $\mathrm{Z}$-vector can produce an accurate wavefunction in the nuclear region of small molecules. In Ref. ${ }^{37}$, we have shown that the Z-vector method can produce very good wavefunction in the nuclear region of moderately heavy molecules like SrF. Similarly, Z-vector method can also produce an accurate wavefunction in the nuclear region of heavy diatomic molecules. ${ }^{38-40}$ So, altogether, we can say that the $\mathrm{Z}$-vector is a very reliable method to produce high quality wavefunction in the core region. In this work, we have also shown that the Z-vector method can produce more accurate results for the magnetic HFS constant than the ECC method and thus, is more reliable for the calculation of various $\mathcal{P}, \mathcal{T}$-odd interaction constants.

\section{Acknowledgments}

Authors acknowledge a grant from CSIR $12^{\text {th }}$ Five Year Plan project on Multi-Scale Simulations of Material 
(MSM) and the resources of the Center of Excellence in Scientific Computing at CSIR-NCL. S.S. and K.T. acknowledge the CSIR for their fellowship. S.P. acknowledges funding from J. C. Bose Fellowship grant of Department of Science and Technology (India).

\section{References}

1. Sakharov A D 1967 JETP Lett. 524

2. Ginges J and Flambaum V 2004 Phys. Rep. 39763

3. Sandars P 1965 Phys. Lett. 14194

4. Sandars P G H 1967 Phys. Rev. Lett. 191396

5. Labzovskii L N 1978 Sov. Phys. JETP 48434

6. Shapiro F L 1968 Phys.-Usp. 11345

7. Pospelov M and Ritz A 2005 Ann. Phys. 318119

8. Knight L Jr, Brom J Jr and Weltner W Jr 1972 J. Chem. Phys. 561152

9. Cizek J 1967 In Advances in Chemical Physics: Correlation Effects in Atoms and Molecules (Hoboken: Wiley)

10. Bartlett R J and Purvis G D 1978 Int. J. Quantum Chem. 14561

11. Monkhorst H J 1977 Int. J. Quantum Chem. 12421

12. Sekino H and Bartlett R J 1984 Int. J. Quantum Chem. 26255

13. Szalay P G, Nooijen M and Bartlett R J 1995 J. Chem. Phys. 103281

14. Arponen J 1983 Ann. Phys. 151311

15. Bishop R, Arponen J and Pajanne P 1989 In Aspects of Many-body Effects in Molecules and Extended Systems (Berlin: Springer-Verlag)

16. Bartlett R J and Noga J 1988 Chem. Phys. Lett. 15029

17. Pal S 1984 Theor. Chim. Acta 66151

18. Kutzelnigg W 1977 In Methods of Electronic Structure Theory (New York: Plenum)

19. Pal S, Prasad M D and Mukherjee D 1983 Theor. Chim. Acta 62523

20. Pal S 1984 Theor. Chim. Acta 66207

21. Tanaka K and Terashima H 1984 Chem. Phys. Lett. 106558
22. Hoffmann M R and Simons J 1988 J. Chem. Phys. 88993

23. Bartlett R J, Kucharski S A and Noga J 1989 Chem. Phys. Lett. 155133

24. Sasmal S, Pathak H, Nayak M K, Vaval N and Pal S 2015 Phys. Rev. A 91022512

25. Handy N C and Schaefer H F 1984 J. Chem. Phys. 815031

26. Salter E A, Trucks G W and Bartlett R J 1989 J. Chem. Phys. 901752

27. Lindgren I and Morrison J 1985 In Atomic Many-Body Theory (New York: Springer-Verlag)

28. DIRAC, a relativistic ab initio electronic structure program, Release DIRAC10 (2010), written by Saue T, Visscher L and Jensen $\mathrm{H} J$, with contributions from Bast R, Dyall K G, Ekström U, Eliav E, Enevoldsen T, Fleig T, Gomes A S P, Henriksson J, Iliaš M, Jacob Ch R, Knecht S, Nataraj H S, Norman P, Olsen J, Pernpointner M, Ruud K, Schimmelpfennig B, Sikkema J, Thorvaldsen A, Thyssen J, Villaume S and Yamamoto S (see http:// www.diracprogram.org)

29. Visscher L and Dyall K 1997 At. Data Nucl. Data Tables 67207

30. Faegri K Jr and Dyall K G 2007 In Introduction to Relativistic Quantum Chemistry (USA: Oxford University Press)

31. Dunning T H 1989 J. Chem. Phys. 901007

32. Dyall K G 2009 J. Phys. Chem. A 11312638

33. http://ccbdb.nist.gov/

34. Quiney H M and Belanzoni P 2002 Chem. Phys. Lett. 353253

35. Knight L B Jr and Weltner W Jr 1971 J. Chem. Phys. 543875

36. Knight L Jr, Easley W, Weltner W Jr and Wilson M 1971 J. Chem. Phys. 54322

37. Sasmal S, Pathak H, Nayak M K, Vaval N and Pal S 2015 Phys. Rev. A 91030503

38. Sasmal S, Pathak H, Nayak M K, Vaval N and Pal S 2015 J. Chem. Phys. 143084119

39. Sasmal S, Pathak H, Nayak M K, Vaval N and Pal S 2016 J. Chem. Phys. 144124307

40. Sasmal S, Pathak H, Nayak M K, Vaval N and Pal S 2016 Phys. Rev. A 93062506 